\title{
Neutron stars and strange stars
}

\author{
Dorota Gondek \\ N. Copernicus Astronomical Center, Polish Academy of Sciences, \\ Bartycka 18, 00-716 Warszawa, Poland
}

\section{Introduction}

If strange quark plasma is the real ground state of baryonic matter (Witten 1984), then some of neutron stars (NS) could actually be strange stars (SS). It is difficult to distinguish SS from NS observationally. They have similar radii and masses and their crusts are built of the same matter. It seems that a good method for testing the existence of SS would be the studies of phenomena related to the stellar pulsations. In 1976 Boriakoff proposed that radial oscillations of NS could be observed within radio subpulses of pulsars. While various modes of pulsations of NS were studied by a number of authors, little attention was paid to seismological signatures of SS. The radial oscilations of bare SS were studied by Väth \& Chanmugam (1992). Recently Weber (this volume) studied properties of stars made of matter described by BPS equation of state (EOS) (Baym et al. 1971) with a ball of strange matter inside, but they mainly concentrated on stability of white-dwarf-like SS. In this work I present fully relativistic calculations of the radial oscillation frequencies of SS. I determined the fundamental frequency for bare SS and SS with two different types of crusts depending on origin (Alcock et al. 1986) of SS and showed differences between them.

\section{Calculations and results}

It is not clear how a crust can form on a SS. If a bare SS is formed in a supernova explosion, surrounding material falls back on it. In this case crust should be described by equation of state of an accreting neutron star (Haensel \& Zdunik 1990 ). If SS are created by converting the nucleus of NS to strange matter than new born SS is bare or have crust built of cold catalyzed matter described by BPS EOS. Independently on the origin, maximum density at the bottom of the crust cannot exceed $\rho_{\text {drip }}$ (Alcock et al. 1986). In Fig. 1 the mass-radius relation is shown for a family of SS and SS with two types of crusts. The EOS of an accreting matter is stiffer and the neutron drip occurs at higher density $\left(6.2 \cdot 10^{11} \mathrm{~g} / \mathrm{cm}^{3}\right)$ compared to the catalyzed EOS $\left(4.3 \cdot 10^{11} \mathrm{~g} / \mathrm{cm}^{3}\right)$. The strange matter is described in the framework of the bag model $P=\left(\rho-\rho_{0}\right) c^{2} / 3$, where $\rho_{0}=4.3 \cdot 10^{14} \mathrm{~g} / \mathrm{cm}^{3}$.

For given EOS of SS I studied the linear, adiabatic, radial, relativistic oscillations (Gondek 1996). The results are shown in Fig. 1 (right). The fundamental frequencies are almost identical for all kind of SS with high central densities, higher than $\rho_{\mathrm{c}}$ of object with $\omega_{\max }$. The differences are mainly for low $\rho_{\mathrm{c}}$, where 

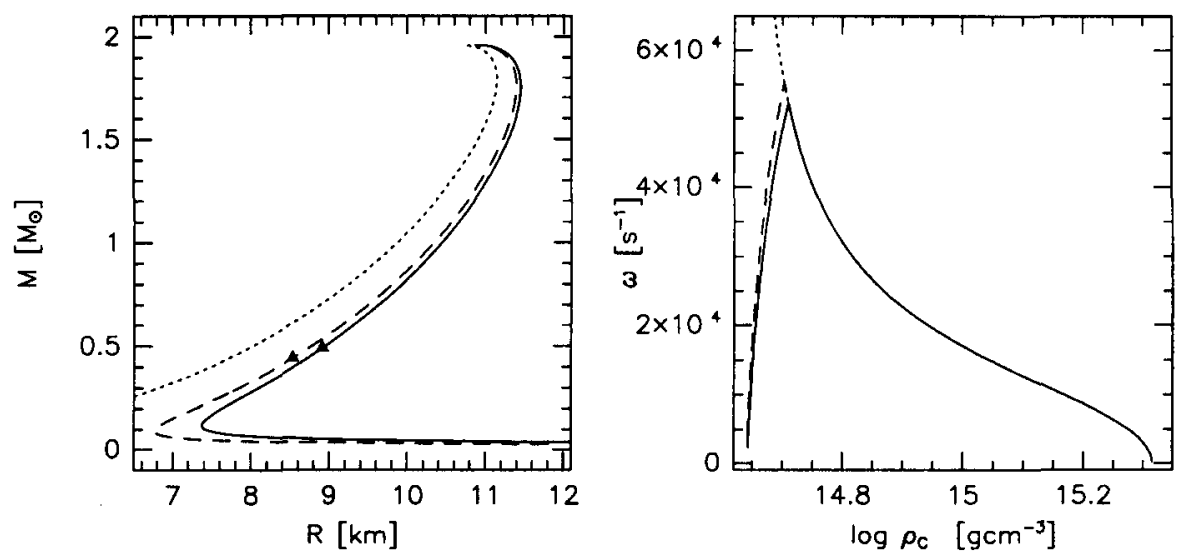

Figure 1. left: Mass vs. radius for bare SS (dotted line), SS with BPS crust (dashed line) and crust built of an accreting matter (solid line) right: corresponding to them fundamental frequencies vs. central density. Triangles correspond to objects with $\omega_{\max }$.

the frequencies of SS with crust are determined by crust pulsations (Gondek 1996).

The oscillation frequencies of bare SS have qualitatively a different dependence on central density compared to NS and SS with crust. In contrast to the NS and SS with crust, the bare SS do not have the largest possible fundamental eigenfrequency (dotted line). Their frequencies go to infinity when the $\rho_{c}$ of the SS approaches its smallest possible value $\left(\rho_{0}\right)$ (Väth \& Chanmugam 1992).

The fundamental frequencies for SS with crust built of an accretion matter are lower than those for SS with crust of catalyzed matter. They are 2, 1.2 and 1.00003 times lower for $\rho_{\mathrm{c}}=4.4,5,6\left[10^{14} \mathrm{~g} / \mathrm{cm}^{3}\right]$ respectively.

Acknowledgments. I am grateful to J. L. Zdunik and R. Moderski for collaboration and for critical suggestions. This work was supported in part by the Polish Committee for Scientific Research (KBN) grants No. 2P30401407, 2P03D01009 and by the program Réseau Formation Recherche of the French Ministère de l'Enseignement Supérieur et de la Recherche.

\section{References}

Alcock, C., Farhi E., Olinto A., 1986, ApJ, 310, 261

Baym G., Pethick C., Sutherland P., 1971, ApJ, 170, 299

Boriakoff V., 1976, ApJ 208, L43

Haensel P., Zdunik, J. L., 1990, A\&A, 229, 117

Gondek D., 1996, in preparation

Witten, E. 1984, Phys. Rev. D 30, 272

Väth H.M., Chanmugan G., 1992, A\&A, 260, 250 